\title{
Copper-catalyzed arylation of alkyl halides with arylaluminum reagents
}

\author{
Bijay Shrestha and Ramesh Giri ${ }^{*}$
}

\author{
Full Research Paper \\ Address: \\ Department of Chemistry \& Chemical Biology, The University of New \\ Mexico, Albuquerque, NM 87131, USA \\ Email: \\ Ramesh Giri* - rgiri@unm.edu \\ * Corresponding author \\ Keywords: \\ alkylation; arylalkanes; copper; cross-coupling; organoaluminum
}

\author{
Beilstein J. Org. Chem. 2015, 11, 2400-2407. \\ doi:10.3762/bjoc. 11.261 \\ Received: 16 September 2015 \\ Accepted: 12 November 2015 \\ Published: 02 December 2015 \\ This article is part of the Thematic Series "Copper catalysis in organic \\ synthesis". \\ Guest Editor: S. R. Chemler
}

(C) 2015 Shrestha and Giri; licensee Beilstein-Institut.

License and terms: see end of document.

\begin{abstract}
We report a $\mathrm{Cu}$-catalyzed coupling between triarylaluminum reagents and alkyl halides to form arylalkanes. The reaction proceeds in the presence of $N, N, N^{\prime}, N^{\prime}$-tetramethyl-o-phenylenediamine $(\mathrm{NN}-1)$ as a ligand in combination with $\mathrm{CuI}$ as a catalyst. This catalyst system enables the coupling of primary alkyl iodides and bromides with electron-neutral and electron-rich triarylaluminum reagents and affords the cross-coupled products in good to excellent yields.
\end{abstract}

\section{Introduction}

Cross-coupling reactions represent one of the most important transformation for carbon-carbon $(\mathrm{C}-\mathrm{C})$ bond formation in organic synthesis [1-9]. These reactions, typically catalyzed by $\mathrm{Pd}$ and $\mathrm{Ni}$, exploit a wide range of organometallic reagents of $\mathrm{Mg}, \mathrm{Zr}, \mathrm{Zn}, \mathrm{Sn}, \mathrm{Al}, \mathrm{B}, \mathrm{Si}$ and $\mathrm{In}$ as sources of nucleophiles. Among these metals/non-metals, $\mathrm{Al}$ offers a unique feature due to its high chemoselectivity and Lewis acidity [10-12]. In addition, $\mathrm{Al}$ also has low toxicity and is an inexpensive and earthabundant metal. Organoaluminum reagents can be prepared directly from metallic aluminum [13-15], which further highlights the potential scope of these reagents in organic synthesis. However, despite extensive investigations and applications of organometallic reagents of $\mathrm{Si}, \mathrm{B}, \mathrm{Mg}, \mathrm{Zn}$ and $\mathrm{Sn}$ in crosscoupling, the utility of organometallic complexes of Al are limited [13,14,16-21]. In many cases, direct transmetalation of organoalanes to $\mathrm{Pd}$ are sluggish and require $\mathrm{ZnCl}_{2}$ or $\mathrm{CdCl}_{2}$ to facilitate reactions through sequential transmetalations [19]. In some cases, intramolecular coordination to Al also enables the couplings of alkylalanes with organo halides [21]. Knochel [15] and Hoveyda [22] have also shown that organoaluminum reagents are capable of transmetalating to $\mathrm{Cu}$-salts. Inspired by these literature reports and our recent investigations, we envisioned that organoaluminum reagents could participate as nucleophile sources in $\mathrm{Cu}$-catalyzed cross-coupling reactions. 
In this artcle, we show that triarylaluminum reagents are excellent coupling partners for $\mathrm{Cu}$-catalyzed cross-coupling reactions. The reaction proceeds for the coupling with primary alkyl iodides and bromides in good to excellent yields.

\section{Results and Discussion}

Recently, we [23-27] and others [10,28-39] reported efficient cross-couplings of oganometallic reagents of $\mathrm{Si}, \mathrm{B}, \mathrm{In}, \mathrm{Zr}, \mathrm{Zn}$, $\mathrm{Mg}$ and $\mathrm{Sn}$ with organo halides [40,41]. Under our reaction conditions, a catalyst derived from the combination of $\mathrm{CuI}$ and 2-(diphenylphosphino)- $N, N$-dimethylaniline (PN-1) remains highly effective for coupling many of these organometallic reagents with aryl halides. In order to expand the scope of our coupling reactions, we utilized the standard condtions for the reaction of commercially available $\mathrm{Ph}_{3} \mathrm{Al}$ with 1-iodooctane using $1 \mathrm{~mol} \%$ each of $\mathrm{CuI}$ and $\mathrm{PN}-1$. However, the product, 1 -phenyloctane (3), was formed only in $34 \%$ yield (Table 1 , entry 1). Further optimization of the reaction conditions revealed that the coupling proceeded in $66 \%$ GC yield when the reaction was performed in NMP using 1 equivalent of $\mathrm{Cs}_{2} \mathrm{CO}_{3}$ as a base and 3 equivlents of $\mathrm{LiCl}$ as an additive in the absence of a ligand (Table 1, entry 2). We then screened a variety of ligands (Scheme 1) and found that $N, N, N^{\prime}, N^{\prime}$-tetramethyl-ophenylenediamine $(\mathrm{NN}-1)$ was an efficient ligand for $\mathrm{CuI}$ that enabled us to increase the product yield to $81 \%$ GC yields $(76 \%$ isolated, Table 1, entry 3) [10,35,42-44]. Reactions containing other PN- and NN-based ligands that are analogous to PN-1 and NN-1 (Scheme 1) afforded cross-coupled product 3 in lower yields than the reaction performed in the absence of NN-1. Reactions containing the bisphosphine ligand, $o$-bis(diphenylphosphine)benzene (PP) and anionic ligands such as 8-hydroxyquinoline (NO) and 2,2,6,6-tetramethyl-3,5-heptanedione (OO, Scheme 1) also formed the product 3 in lower yields and the reaction performed in the absence of NN-1. The reaction does not proceed in the absence of $\mathrm{CuI}$ (Table 1, entry 4). The crosscoupled product 3 is formed in 50\% and 54\% yield, respectively, in the absence of $\mathrm{LiCl}$ and $\mathrm{Cs}_{2} \mathrm{CO}_{3}$ (Table 1, entries 5 and 6). The reacton with 2 and 4 equivalents of $\mathrm{LiCl}$ also afforded product 3 in comparable yields ( $78 \%$ and $76 \%$, respectively) to that of the standard reaction (Table 1, entries 7 and 8). However, excess of $\mathrm{LiCl}$ was found to be detrimental to the reaction (Table 1, entry 9). The reaction could also be performed at a temperature as low as $80^{\circ} \mathrm{C}$ affording the coupled product 3 only in slightly lower yields than that of the standard reaction (Table 1, entries 10 and 11).

After establishing the combination of $\mathrm{NN}-1$ and $\mathrm{CuI}$ as the best catalyst, we began to explore the substrate scope of the reaction. While the reaction proceeded in good yields with alkyl iodides (Table 2, entries 1-3) by using $1 \mathrm{~mol} \%$ of the catalyst, reactions with alkyl bromides, which are more readily available and less expensive than alkyl iodides, required $10 \mathrm{~mol} \%$ of $\mathrm{NN}-1 / \mathrm{CuI}$ (Table 2, entries 4-15). The reaction can be performed with electron-neutral and electron-rich triarylaluminum reagents [45]. The reaction tolerates a variety of functional groups on alkyl halides including highly sensitive esters

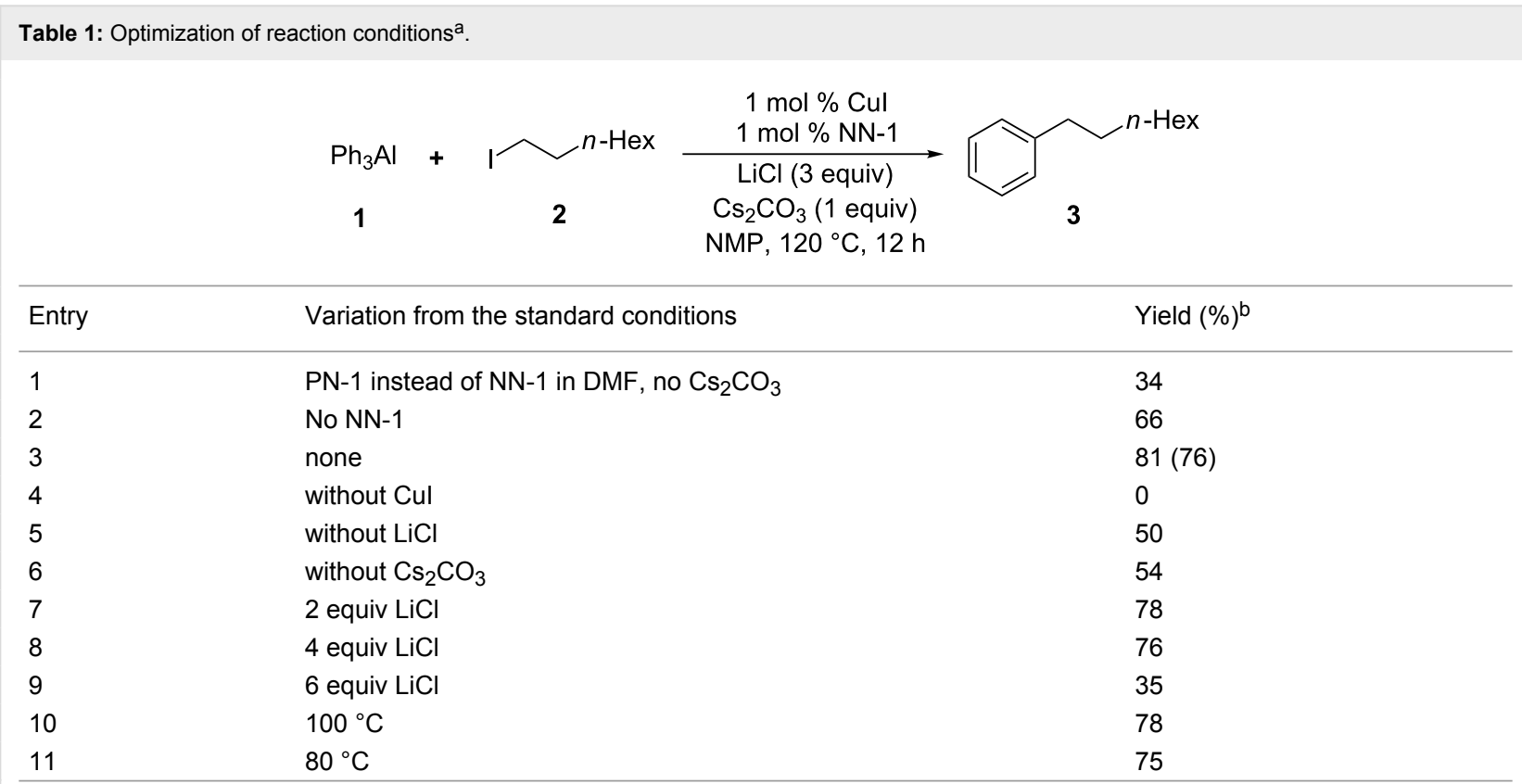

aReactions were run in $0.5 \mathrm{~mL}$ DMF. Commercially available $\mathrm{Ph}_{3} \mathrm{Al}$ was used. ${ }^{\mathrm{b}} \mathrm{GC}$ yields (average of at least two parallel runs) calibrated against 2-nitrobiphenyl as an internal standard. Value in parenthesis is the isolated yield $(1.0 \mathrm{mmol})$. 
<smiles>Cc1ccccc1Pc1ccccc1</smiles>

$\mathrm{PN}-1,50 \%$<smiles>CC(C)Pc1ccccc1-c1ccccc1N(C)C</smiles>

PN-6, 37\%

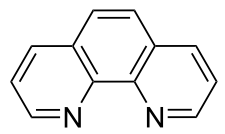

NN-3, 39\%<smiles>Pc1ccccc1N1CCCCC1</smiles>

PN-2, 55\%<smiles>Pc1ccccc1P</smiles>

PN-7, 27\%

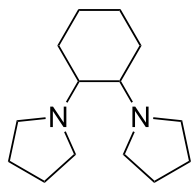

NN-4, 45\%<smiles>Pc1ccccc1N1CCOCC1</smiles>

PN-3, 35\%<smiles>c1ccc(P=Nc2ccc(N=Pc3ccccc3)cc2)cc1</smiles>

PN-8, 59\%<smiles>Pc1cccc(Pc2ccccc2)c1P</smiles>

PP, $26 \%$<smiles>c1ccc(-c2cccc3cccnc23)cc1</smiles>

PN-4, 32\%<smiles>CN(C)c1ccccc1N(C)C</smiles>

$\mathrm{NN}-1,81 \%$<smiles>Oc1cccc2cccnc12</smiles>

$t-\mathrm{Bu}$<smiles>CN(C)c1cccc2cccc(N(C)C)c12</smiles>

NN-2, 45\%

Scheme 1: Ligands used for reaction optimization.

(Table 2, entries, 5, 9 and 11), nitriles (Table 2, entries 6 and 7) and olefins (Table 2, entries 4, 8, 10, 13 and 15) [46]. With $10 \mathrm{~mol} \%$ catalyst loading, the reaction can also be extended to the coupling of triarylaluminum reagents with benzyl bromides (Table 2, entries 12 and 14) [43].
Based on literature reports and our recent mechanistic work on $\mathrm{Cu}$-catalyzed cross-couplings [23-25], we propose a catalytic cycle for the current reaction (Scheme 2). It is evident from the optimization of reaction conditions that both $\mathrm{NN}-1$ and $\mathrm{LiCl}$ improve product yields for the current coupling of triarylalu-

Table 2: Coupling of triarylaluminum reagents with alkyl iodides and bromides ${ }^{a}$.

$$
\begin{aligned}
& \mathrm{Ar}_{3} \mathrm{Al}+\mathrm{Alkyl}-\mathrm{I}, \mathrm{Br} \underset{[\mathrm{LiCl}(3 \text { equiv })], \mathrm{Cs}_{2} \mathrm{CO}_{3}(1 \text { equiv) }}{\stackrel{1 \text { or } 10 \mathrm{~mol} \% \mathrm{Cul}}{\longrightarrow}} \mathrm{Alkyl-Ar} \\
& \text { NMP }, 120^{\circ} \mathrm{C}, 12 \mathrm{~h}
\end{aligned}
$$

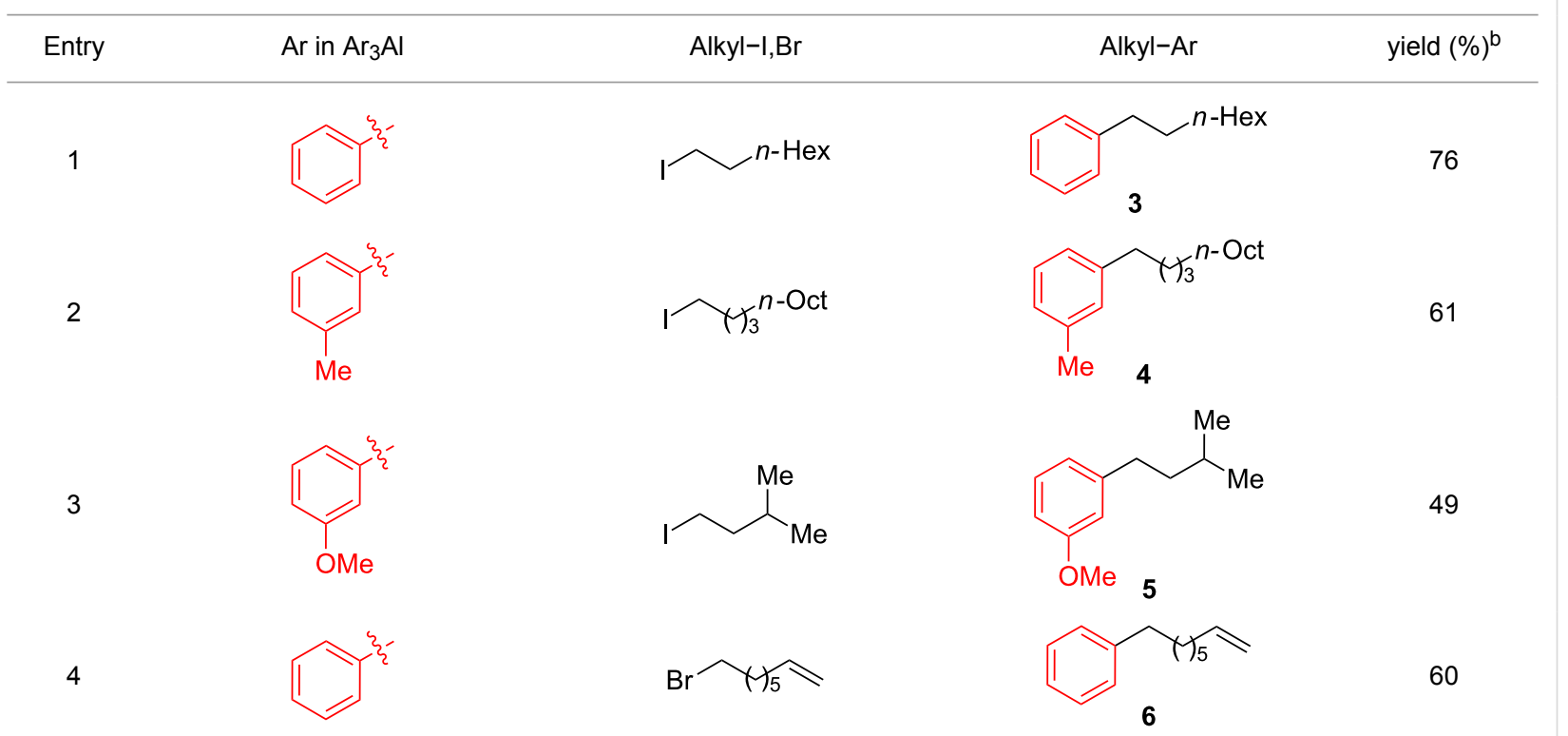


Table 2: Coupling of triarylaluminum reagents with alkyl iodides and bromides ${ }^{\mathrm{a}}$. (continued)

5<smiles>Ic1ccccc1</smiles>

6<smiles>Cc1ccccc1</smiles>

7<smiles>Cc1cccc(I)c1</smiles>

8<smiles>Cc1cccc(I)c1</smiles>

9<smiles>Cc1cccc(I)c1</smiles>

10<smiles>Cc1ccccc1I</smiles>

11<smiles>Cc1ccccc1I</smiles>

12<smiles>COc1cccc(I)c1</smiles>

13<smiles>COc1cccc([Tl])c1</smiles>

14<smiles>COc1ccccc1I</smiles>

15

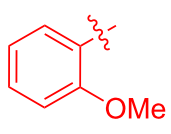<smiles>CCOC(=O)[14CH2]CBr</smiles><smiles>CC(C)(C)C(C#N)CBr</smiles><smiles>CC(C)(C#N)CCBr</smiles><smiles>C=C[14CH2]CBr</smiles><smiles>CCOC(=O)[14CH2]CBr</smiles><smiles>C=C[14CH2]CBr</smiles><smiles>CCOC(=O)[14CH2]CBr</smiles><smiles>BrCc1ccccc1</smiles><smiles>C=C[14CH2]CBr</smiles><smiles>BrCc1ccccc1</smiles>

$\mathrm{Br}^{\mathrm{H}_{2}} \mathrm{\sim}$<smiles>CCOC(=O)NCc1ccccc1</smiles><smiles>CC(C)(C)CCc1ccccc1</smiles><smiles>Cc1cccc(CCC(C)(C)C#N)c1</smiles><smiles>C=CCCc1cccc(C)c1</smiles><smiles>CCOC(=O)[AlH2]Cc1cccc(C)c1</smiles><smiles>C=CCCc1ccccc1C</smiles>

12<smiles>CCOC(=O)NCc1ccccc1C</smiles>

13<smiles>COc1cccc(Cc2ccccc2)c1</smiles>

14<smiles>C=C[14CH2]Cc1cccc(OC)c1</smiles><smiles>COc1ccccc1Cc1ccccc1</smiles>

16<smiles>C=CCCc1ccccc1OC</smiles>

17

aReactions were run in $5 \mathrm{~mL}$ DMF. Reactions for entries $1-3$ were run with $1 \mathrm{~mol} \% \mathrm{NN}-1 / \mathrm{Cul}$. Reactions for entries $4-15$ were run with $10 \mathrm{~mol} \%$ $\mathrm{NN}-1 / \mathrm{Cul}$. Triarylaluminum reagents, except the commercially available $\mathrm{Ph}_{3} \mathrm{Al}$, were prepared from the reaction of 3 equivalents of ArLi reagents with $\mathrm{AlCl}_{3}(99.99 \%$ purity) in THF at room temperature and were used without further purification. Each reaction contains 3 equivalents of LiCl, written in parenthesis below the reaction arrow, which is generated during the preparation of triarylaluminum reagents. bYields are for products isolated by column chromatography from a $1.0 \mathrm{mmol}$ scale reaction. 
minum reagents with alkyl halides (Table 1). As such, we believe that organoaluminate complexes such as $\mathbf{1 8}$, generated from the binding of $\mathrm{LiCl}$ to three-coordinate triarylaluminum reagents, are the actual species in solution that undergo transmetalation with $\mathrm{NN}$-bound $\mathrm{CuX}(\mathrm{X}=\mathrm{I}, \mathrm{Br})$ to generate (NN)CuAr complexes as the reaction intermediates. Catalytically competent $\mathrm{Cu}^{\mathrm{I}}$-complexes that contain nitrogen-based ligands have previously been synthesized and fully characterized structurally [47-51]. Triorganoaluminum complexes are also known to form triorganoaluminate species in the presence of anions in solution [52-57]. In addition, organoaluminum reagents have been demonstrated to undergo transmetalation with $\mathrm{Cu}$ salts based on their participation in allylic and conjugate addition reactions $[11,15,43]$. Similar $\mathrm{Cu}$-catalyzed couplings of organometallic reagents with alkyl electrophiles have previously been shown to proceed via an $\mathrm{S}_{\mathrm{N}} 2$ process $[34,35]$. Therefore, we believe that a similar mechanistic scenario can also be envisioned in the current $\mathrm{Cu}$-catalyzed cross-coupling of triarylaluminum reagents with primary alkyl halides that involves $(\mathrm{NN}) \mathrm{CuAr}$ as the reaction intermediates.

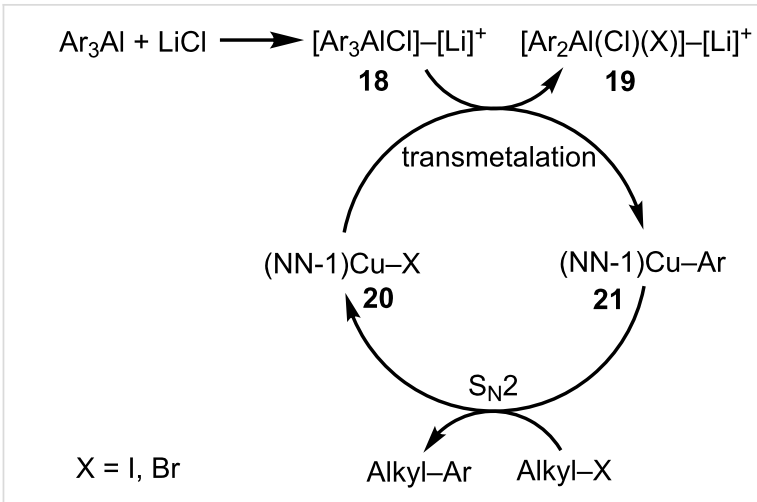

Scheme 2: Proposed catalytic cycle.

\section{Conclusion}

In summary, we have developed a $\mathrm{Cu}$-catalyzed coupling of triarylaluminum reagents with primary alkyl iodides and bromides. The reaction proceeds in the presence of $\mathrm{NN}-1 / \mathrm{CuI}$ as an effective catalyst. Electron-neutral and electron-rich triarylaluminum reagents can be coupled with a variety of alkyl iodides and bromides containing a range of sensitive functional groups such as olefins, nitriles and esters, affording the alkylated arenes in good to excellent yields.

\section{Experimental}

General information. All the reactions and handling of chemicals were done inside a nitrogen-filled glovebox unless stated otherwise. All glassware were dried in an oven before use. All commercial reagents were used as received without further purification. Anhydrous solvents and triphenylaluminum were purchased from Sigma-Aldrich. Pure triarylaluminum reagents other than $\mathrm{Ph}_{3} \mathrm{Al}$ were synthesized following the reported procedure [56]. Ligands PN-5, PN-6, NN-2, NN-3, PP, NO and $\mathrm{OO}$ were purchased from commercial sources. Ligands $\mathrm{PN}-1$, PN-2, PN-3, PN-4, PN-7 [58], PN-8, NN-1 [59], and NN-4 [60] were synthesized following the reported procedures [61]. ${ }^{1} \mathrm{H}$ and ${ }^{13} \mathrm{C}$ NMR spectra were recorded on a Bruker instrument (300 and $75 \mathrm{MHz}$, respectively) and internally referenced to the residual solvent signals of $\mathrm{CDCl}_{3}$ at 7.26 and at $77.16 \mathrm{ppm}$, respectively.

General procedure for cross-coupling. To a suspension of $\mathrm{AlCl}_{3}(133.3 \mathrm{mg}, 1.0 \mathrm{mmol})$ in THF $(2 \mathrm{~mL})$ was added dropwise a solution of aryllithium $(3.0 \mathrm{mmol}$, generated from the lithiation of aryl iodides with 1 equiv of $n$-BuLi in THF) at room temperature. After 45 minutes, the solvent was removed to obtain a triarylaluminum reagent containing 3 equivalents of $\mathrm{LiCl}$, which was then dissolved in NMP $(5 \mathrm{~mL})$. Alkyl halide (1.0 mmol), CuI (1.9 mg, $0.010 \mathrm{mmol}$, for alkyl iodides; $19.0 \mathrm{mg}, 0.10 \mathrm{mmol}$, for alkyl bromides) and NN-1 (1.6 mg, $0.010 \mathrm{mmol}$, for alkyl iodides; $16.4 \mathrm{mg}, 0.10 \mathrm{mmol}$, for alkyl bromides) were then added to the solution of the triarylaluminum reagent. The reaction mixture was then tightly capped, taken out of the glovebox, placed in an oil bath pre-heated to $120^{\circ} \mathrm{C}$ and vigorously stirred. After $12 \mathrm{~h}$, the reaction mixture was cooled to room temperature, diluted with ethyl acetate $(15 \mathrm{~mL})$ and washed with $\mathrm{H}_{2} \mathrm{O}(5 \mathrm{~mL} \times 3)$. The aqueous fraction was extracted back with ethyl acetate $(5 \mathrm{~mL} \times 3)$ and combined with the first ethyl acetate fraction. The combined ethyl acetate fractions were dried over $\mathrm{Na}_{2} \mathrm{SO}_{4}$ and the solvent was removed on a rotary evaporator. The product was purified by silica gel column chromatography using $0-5 \%$ ethyl acetate in hexanes.

$\boldsymbol{n}$-Octylbenzene (3) [62]: The title compound $\mathbf{3}$ was obtained as a colorless oil (144 mg, 76\% yield) after purification by silica gel column chromatography. ${ }^{1} \mathrm{H} \mathrm{NMR}\left(300 \mathrm{MHz}, \mathrm{CDCl}_{3}\right) \delta$ $0.89(\mathrm{t}, J=6.6 \mathrm{~Hz}, 3 \mathrm{H}), 1.29-1.32(\mathrm{~m}, 10 \mathrm{H}), 1.58-1.68(\mathrm{~m}$, 2H), $2.62(\mathrm{~m}, J=8.1 \mathrm{~Hz}, 2 \mathrm{H}), 7.16-7.21(\mathrm{~m}, 3 \mathrm{H}), 7.27-7.32$ $(\mathrm{m}, 2 \mathrm{H}) ;{ }^{13} \mathrm{C} \mathrm{NMR}\left(75 \mathrm{MHz}, \mathrm{CDCl}_{3}\right) \delta 14.2,22.8,29.4,29.5$, 29.6, 31.7, 32.0, 36.1 125.7, 128.3, 128.5, 143.1; GC-MS (m/z) 190.1 .

1-Dodecyl-3-methylbenzene (4): The title compound 4 was obtained as yellow oil (159 mg, 61\% yield) after purification by silica gel column chromatography. ${ }^{1} \mathrm{H} \mathrm{NMR}\left(300 \mathrm{MHz}, \mathrm{CDCl}_{3}\right)$ $\delta 0.90(\mathrm{t}, J=6.3 \mathrm{~Hz}, 3 \mathrm{H}), 1.28-1.31(\mathrm{~m}, 18 \mathrm{H}), 1.53-1.64(\mathrm{~m}$, $2 \mathrm{H}), 2.35(\mathrm{~s}, 3 \mathrm{H}), 2.55-2.60(\mathrm{~m}, 2 \mathrm{H}), 6.99-7.02(\mathrm{~m}, 3 \mathrm{H}), 7.18$ $(\mathrm{t}, J=6.9 \mathrm{~Hz}, 1 \mathrm{H}) ;{ }^{13} \mathrm{C} \mathrm{NMR}\left(75 \mathrm{MHz}, \mathrm{CDCl}_{3}\right) \delta 14.3,21.6$, 22.9, 27.1, 29.5, 29.6, 29.7, 29.8, 31.7, 32.1, 36.1, 45.3, 125.5, 126.4, 128.3, 129.4, 137.9, 143.1; GC-MS ( $\mathrm{m} / \mathrm{z}) 260.1$. 
1-Isopentyl-3-methoxybenzene (5): The title compound 5 was obtained as colorless oil ( $87 \mathrm{mg}, 49 \%$ yield) after purification by silica gel column chromatography. ${ }^{1} \mathrm{H}$ NMR $(300 \mathrm{MHz}$, $\left.\mathrm{CDCl}_{3}\right) \delta 0.93(\mathrm{~s}, 6 \mathrm{H}), 1.46-1.64(\mathrm{~m}, 3 \mathrm{H}), 2.6(\mathrm{t}, J=7.8 \mathrm{~Hz}$, $2 \mathrm{H}), 3.80(\mathrm{~s}, 3 \mathrm{H}), 6.71-6.80(\mathrm{~m}, 2 \mathrm{H}), 7.12-7.38(\mathrm{~m}, 2 \mathrm{H})$; ${ }^{13} \mathrm{C}$ NMR $\left(75 \mathrm{MHz}, \mathrm{CDCl}_{3}\right) \delta 22.7,27.8,33.9,40.8,55.2$, 110.9, 114.3, 120.9, 129.3, 144.9, 159.7; GC-MS (m/z) 178.1.

7-Octen-1-ylbenzene (6) [63]: The title compound 6 was obtained as a colorless oil (113 mg, 60\% yield) after purification by silica gel column chromatography. ${ }^{1} \mathrm{H}$ NMR $(300 \mathrm{MHz}$, $\left.\mathrm{CDCl}_{3}\right) \delta 1.33-1.41(\mathrm{~m}, 6 \mathrm{H}), 1.60-1.65(\mathrm{~m}, 2 \mathrm{H}), 2.00-2.08(\mathrm{~m}$, $2 \mathrm{H}), 2.61(\mathrm{t}, J=7.5 \mathrm{~Hz}, 2 \mathrm{H}), 4.91-5.03(\mathrm{~m}, 2 \mathrm{H}), 5.75-5.88(\mathrm{~m}$, 1H), 7.17-7.20 (m, 3H), 7.25-7.31 (m, 2H); ${ }^{13} \mathrm{C}$ NMR $(75$ $\left.\mathrm{MHz}, \mathrm{CDCl}_{3}\right) \delta 28.9,29.1,29.2,31.5,33.9,36.0,114.3,125.6$, $128.3,128.5,139.2,142.9$; GC-MS $(m / z) 188.1$.

Ethyl 5-phenylvalerate (7) [64]: The title compound 7 was obtained as yellow oil (120 mg, 58\% yield) after purification by silica gel column chromatography. ${ }^{1} \mathrm{H}$ NMR $\left(300 \mathrm{MHz}, \mathrm{CDCl}_{3}\right)$ $\delta 1.26(\mathrm{t}, J=9.0 \mathrm{~Hz}, 3 \mathrm{H}), 1.66-1.70(\mathrm{~m}, 4 \mathrm{H}), 2.31-2.35(\mathrm{~m}$, $2 \mathrm{H}), 2.62-2.67(\mathrm{~m}, 2 \mathrm{H}), 4.13(\mathrm{q}, J=6.9 \mathrm{~Hz}, 2 \mathrm{H}), 7.17-7.22(\mathrm{~m}$, $3 \mathrm{H}), 7.26-7.32(\mathrm{~m}, 2 \mathrm{H}) ;{ }^{13} \mathrm{C} \mathrm{NMR}\left(75 \mathrm{MHz}, \mathrm{CDCl}_{3}\right) \delta 14.3$, 24.7, 33.5, 34.2, 35.6, 60.3, 125.8, 128.4, 128.4, 142.2, 173.7; GC-MS $(m / z) 206.1$.

2,2-Dimethyl-6-phenylhexanenitrile (8) [65]: The title compound 8 was obtained as yellow oil (143 mg, 71\% yield) after purification by silica gel column chromatography. ${ }^{1} \mathrm{H}$ NMR $\left(300 \mathrm{MHz}, \mathrm{CDCl}_{3}\right) \delta 1.34(\mathrm{~s}, 6 \mathrm{H}), 1.54-1.58(\mathrm{~m}, 4 \mathrm{H})$, 1.62-1.72 (m, 2H), 2.63-2.68 (m, 2H), 7.17-7.23 (m, 3H), 7.26-7.33 (m, 2H); ${ }^{13} \mathrm{C} \mathrm{NMR}\left(75 \mathrm{MHz}, \mathrm{CDCl}_{3}\right) \delta 25.0,26.7$, $31.5,32.4,35.7,40.9,125.2,125.8,128.4,142.2$; GC-MS $(\mathrm{m} / \mathrm{z})$ 201.1 .

2,2-Dimethyl-6-(3-methylphenyl)hexanenitrile (9): The title compound 9 was obtained as yellow oil (189 mg, 88\% yield) after purification by silica gel column chromatography. ${ }^{1} \mathrm{H}$ NMR $\left(300 \mathrm{MHz}, \mathrm{CDCl}_{3}\right) \delta 1.35(\mathrm{~s}, 6 \mathrm{H}), 1.55-1.59(\mathrm{~m}, 4 \mathrm{H})$, $1.63-1.72(\mathrm{~m}, 2 \mathrm{H}), 2.36(\mathrm{~s}, 3 \mathrm{H}), 2.61-2.67$ (m, 2H), 6.99-7.02 $(\mathrm{m}, 3 \mathrm{H}), 7.19(\mathrm{t}, J=3.9 \mathrm{~Hz}, 1 \mathrm{H}) ;{ }^{13} \mathrm{C} \mathrm{NMR}\left(75 \mathrm{MHz}, \mathrm{CDCl}_{3}\right) \delta$ $21.4,25.0,26.6,31.5,32.3,35.6,41.3,125.2,125.3,126.5$, 128.2, 129.2, 137.8, 142.1; GC-MS (m/z) 215.1.

1-(6-Hepten-1-yl)-3-methylbenzene (10): The title compound 10 was obtained as colorless oil (100 mg, 53\% yield) after purification by silica gel column chromatography. ${ }^{1} \mathrm{H}$ NMR $\left(300 \mathrm{MHz}, \mathrm{CDCl}_{3}\right) \delta 1.34-1.45(\mathrm{~m}, 4 \mathrm{H}), 1.59-1.66(\mathrm{~m}, 2 \mathrm{H})$, 2.02-2.09 (m, 2H), $2.33(\mathrm{~s}, 3 \mathrm{H}), 2.57(\mathrm{t}, J=7.8 \mathrm{~Hz}, 2 \mathrm{H})$, 4.92-5.03 (m, 2H), 5.75-5.88 (m, 1H), 6.97-7.00 (m, 3H), 7.17 $(\mathrm{t}, J=3.9 \mathrm{~Hz}, 1 \mathrm{H}) ;{ }^{13} \mathrm{C} \mathrm{NMR}\left(75 \mathrm{MHz}, \mathrm{CDCl}_{3}\right) \delta 28.8,29.7$,
$31.4,33.7,35.6,114.2,125.4,126.3,128.1,129.2,137.7,139.1$, 142.8; GC-MS $(\mathrm{m} / \mathrm{z})$ 188.2.

Ethyl 5-(3-methylphenyl)valerate (11) [66]: The title compound 11 was obtained as a yellow oil (130 mg, 59\% yield) after purification by silica gel column chromatography. ${ }^{1} \mathrm{H}$ NMR $\left(300 \mathrm{MHz}, \mathrm{CDCl}_{3}\right) \delta 1.28(\mathrm{t}, J=7.2 \mathrm{~Hz}, 3 \mathrm{H})$, $1.66-1.72(\mathrm{~m}, 4 \mathrm{H}), 2.33-2.37(\mathrm{~m}, 5 \mathrm{H}), 2.60-2.65(\mathrm{~m}, 2 \mathrm{H}), 4.15$ (q, $J=7.2 \mathrm{~Hz}, 2 \mathrm{H}), 6.98-7.04(\mathrm{~m}, 3 \mathrm{H}), 7.18(\mathrm{t}, J=7.5 \mathrm{~Hz}, 1 \mathrm{H})$; ${ }^{13} \mathrm{C} \mathrm{NMR}\left(75 \mathrm{MHz}, \mathrm{CDCl}_{3}\right) \delta 14.3,21.5,24.7,31.0,34.3,35.6$, $60.2,125.4,126.5,128.3,129.3,137.9,142.2,173.7$; GC-MS $(\mathrm{m} / \mathrm{z}) 220.2$.

1-(5-Hexen-1-yl)-2-methylbenzene (12) [67]: The title compound 12 was obtained as colorless oil ( $80 \mathrm{mg}, 46 \%$ yield) after purification by silica gel column chromatography. ${ }^{1} \mathrm{H}$ NMR $\left(300 \mathrm{MHz}, \mathrm{CDCl}_{3}\right) \delta 1.48-1.67(\mathrm{~m}, 4 \mathrm{H}), 2.09-2.16(\mathrm{~m}, 2 \mathrm{H})$, $2.33(\mathrm{~s}, 3 \mathrm{H}), 2.59-2.65(\mathrm{~m}, 2 \mathrm{H}), 4.98-5.07(\mathrm{~m}, 2 \mathrm{H}), 5.77-5.91$ $(\mathrm{m}, 1 \mathrm{H}), 7.11-7.15(\mathrm{~m}, 4 \mathrm{H}) ;{ }^{13} \mathrm{C} \mathrm{NMR}\left(75 \mathrm{MHz}, \mathrm{CDCl}_{3}\right) \delta$ 19.4, 29.1, 29.9, 33.3, 33.8, 114.6, 125.9, 125.9, 128.9, 130.2, 135.9, 139.0, 141.0; GC-MS (m/z) 174.1.

Ethyl 5-(2-methylphenyl)valerate (13) [68]: The title compound 13 was obtained as yellow oil (203 mg, 92\% yield) after purification by silica gel column chromatography. ${ }^{1} \mathrm{H}$ NMR $\left(300 \mathrm{MHz}, \mathrm{CDCl}_{3}\right) \delta 1.26(\mathrm{t}, J=7.2 \mathrm{~Hz}, 3 \mathrm{H}), 1.58-1.76(\mathrm{~m}$, $4 \mathrm{H}), 2.31-2.37(\mathrm{~m}, 5 \mathrm{H}), 2.6-2.65(\mathrm{~m}, 2 \mathrm{H}), 4.13(\mathrm{q}, J=7.2 \mathrm{~Hz}$, 2H), 7.11-7.12 (m, 4H); $\left.{ }^{13} \mathrm{C} \mathrm{NMR} \mathrm{(75} \mathrm{MHz,} \mathrm{CDCl}_{3}\right) \delta 14.4$, 19.4, 25.1, 29.8, 33.1, 34.4, 60.4, 126.0, 128.9, 130.3, 135.9, 140.5, 173.8; GC-MS $(\mathrm{m} / \mathrm{z}) 220.2$.

1-Benzyl-3-methoxybenzene (14) [69]: The title compound 14 was obtained as a light yellow oil (105 mg, 53\% yield) after purification by silica gel column chromatography. ${ }^{1} \mathrm{H}$ NMR $\left(300 \mathrm{MHz}, \mathrm{CDCl}_{3}\right) \delta 2.82(\mathrm{~s}, 3 \mathrm{H}), 3.8(\mathrm{~s}, 2 \mathrm{H}), 6.77-6.84(\mathrm{~m}$, $2 \mathrm{H}), 6.91(\mathrm{t}, J=9.0 \mathrm{~Hz}, 1 \mathrm{H}), 7.20-7.34(\mathrm{~m}, 6 \mathrm{H}) ;{ }^{13} \mathrm{C} \mathrm{NMR}(75$ $\left.\mathrm{MHz}, \mathrm{CDCl}_{3}\right) \delta 42.0,55.2,111.4,114.9,121.4,126.2,128.3$, 128.5, 129.5, 140.9, 142.7, 159.8; GC-MS (m/z) 198.1.

1-Methoxy-3-(oct-7-en-1-yl)benzene (15): The title compound 15 was obtained as colorless oil (113 mg, 52\% yield) after purification by silica gel column chromatography. ${ }^{1} \mathrm{H}$ NMR $\left(300 \mathrm{MHz}, \mathrm{CDCl}_{3}\right) \delta 1.27-1.42(\mathrm{~m}, 4 \mathrm{H}), 1.55-1.65$ (m, 4H), 2.01-2.05 (m, 2H), 2.59 (t, $J=7.5 \mathrm{~Hz}, 2 \mathrm{H}), 3.81$ (s, $3 \mathrm{H}), 4.92-5.02(\mathrm{~m}, 2 \mathrm{H}), 5.75-5.88(\mathrm{~m}, 1 \mathrm{H}), 6.72-7.36(\mathrm{~m}, 4 \mathrm{H})$; ${ }^{13} \mathrm{C}$ NMR $\left(75 \mathrm{MHz}, \mathrm{CDCl}_{3}\right) \delta 29.1,29.3,29.6,31.4,33.9,35.8$, $55.2,110.9,114.3,121.0,129.3,129.8,139.3,144.7$; GC-MS $(\mathrm{m} / \mathrm{z}) 218.2$.

1-Benzyl-2-methoxybenzene (16) [70]: The title compound 16 was obtained as light yellow oil (135 mg, 68\% yield) after 
purification by silica gel column chromatography. ${ }^{1} \mathrm{H}$ NMR $\left(300 \mathrm{MHz}, \mathrm{CDCl}_{3}\right) \delta 3.86(\mathrm{~s}, 3 \mathrm{H}), 4.04(\mathrm{~s}, 2 \mathrm{H}), 6.90-6.96(\mathrm{~m}$, 2H), 7.11-7.14 (m, 1H), 7.21-7.36 (m, 6H); ${ }^{13} \mathrm{C}$ NMR $(75$ $\left.\mathrm{MHz}, \mathrm{CDCl}_{3}\right) \delta 35.9,55.4,110.5,120.6,125.8,127.5,128.3$, 129.0, 129.7, 130.4, 141.1, 157.4; GC-MS (m/z) 198.2.

1-Methoxy-2-(pent-4-en-1-yl)benzene (17) [71]: The title compound 17 was obtained as yellow oil ( $83 \mathrm{mg}, 47 \%$ yield) after purification by silica gel column chromatography. ${ }^{1} \mathrm{H}$ NMR $\left(300 \mathrm{MHz}, \mathrm{CDCl}_{3}\right) \delta 1.64-1.74(\mathrm{~m}, 2 \mathrm{H}), 2.08-2.15$ $(\mathrm{m}, 2 \mathrm{H}), 2.61-2.66(\mathrm{~m}, 2 \mathrm{H}), 3.83(\mathrm{~s}, 3 \mathrm{H}), 5.01-5.08(\mathrm{~m}, 2 \mathrm{H})$, 5.80-5.94 (m, 1H), 6.84-6.92 (m, 3H), $7.15(\mathrm{t}, J=7.5 \mathrm{~Hz}, 1 \mathrm{H})$; ${ }^{13} \mathrm{C}$ NMR $\left(75 \mathrm{MHz}, \mathrm{CDCl}_{3}\right) \delta 29.2,33.8,41.5,55.4,110.4$, $114.5,117.9,120.4,127.0,139.1,145.3,157.2$; GC-MS $(\mathrm{m} / \mathrm{z})$ 176.1 .

\section{Acknowledgments}

We thank the University of New Mexico (UNM) for financial support, and upgrades to the NMR (NSF grants CHE08-40523 and CHE09-46690) and MS Facilities.

\section{References}

1. Heck, R. F. In Comprehensive Organic Synthesis; Trost, B. M.; Fleming, I., Eds.; Pergamon Press: Oxford, 1991; Vol. 4, pp 833 ff.

2. Diederich, F.; Stang, P. J. Metal-Catalyzed Cross-Coupling Reactions; Wiley-VCH: New York, 1998.

3. Negishi, E.-i.; Hu, Q.; Huang, Z.; Qian, M.; Wang, G. Aldrichimica Acta 2005, 38, 71.

4. Fu, G. C. Acc. Chem. Res. 2008, 41, 1555-1564. doi:10.1021/ar800148f

5. Knochel, P.; Jones, P., Eds. Organozinc Reagents: A Practical Approach; Oxford University Press: New York, 1999.

6. Jana, R.; Pathak, T. P.; Sigman, M. S. Chem. Rev. 2011, 111, 1417-1492. doi:10.1021/cr100327p

7. Chang, W.-T. T.; Smith, R. C.; Regens, C. S.; Bailey, A. D.; Werner, N. S.; Denmark, S. E. Org. React. 2011, 75, 213-746.

8. Nakao, Y.; Hiyama, T. Chem. Soc. Rev. 2011, 40, 4893-4901. doi:10.1039/c1cs15122c

9. Sore, H. F.; Galloway, W. R. J. D.; Spring, D. R. Chem. Soc. Rev. 2012, 41, 1845-1866. doi:10.1039/C1CS15181A

10. Tsubouchi, A.; Muramatsu, D.; Takeda, T. Angew. Chem., Int. Ed. 2013, 52, 12719-12722. doi:10.1002/anie.201306882

11. Thaler, T.; Knochel, P. Angew. Chem., Int. Ed. 2009, 48, 645-648. doi:10.1002/anie.200804446

12. Krasovskiy, A.; Malakhov, V.; Gavryushin, A.; Knochel, P. Angew. Chem., Int. Ed. 2006, 45, 6040-6044. doi:10.1002/anie.200601450

13. Huo, S. Org. Lett. 2003, 5, 423-425. doi:10.1021/ol0272693

14. Nicolaou, K. C.; Bulger, P. G.; Sarlah, D. Angew. Chem., Int. Ed. 2005, 44, 4442-4489. doi:10.1002/anie.200500368

15. Blümke, T. D.; Groll, K.; Karaghiosoff, K.; Knochel, P. Org. Lett. 2011, 13, 6440-6443. doi:10.1021/ol202733v

16. Chen, X.; Zhou, L.; Li, Y.; Xie, T.; Zhou, S. J. Org. Chem. 2014, 79, 230-239. doi:10.1021/jo4024123
17. Naka, H.; Uchiyama, M.; Matsumoto, Y.; Wheatley, A. E. H.; McPartlin, M.; Morey, J. V.; Kondo, Y. J. Am. Chem. Soc. 2007, 129, 1921-1930. doi:10.1021/ja064601n

18. Getmanenko, Y. A.; Twieg, R. J. J. Org. Chem. 2008, 73, 830-839. doi:10.1021/jo701812t

19. Koszinowski, K.; Böhrer, P. Organometallics 2009, 28, 771-779. doi:10.1021/om800947t

20. Lipshutz, B. H.; Bülow, G.; Lowe, R. F.; Stevens, K. L. Tetrahedron 1996, 52, 7265-7276. doi:10.1016/0040-4020(96)00250-5

21. Blum, J.; Gelman, D.; Baidossi, W.; Shakh, E.; Rosenfeld, A.; Aizenshtat, Z.; Wassermann, B. C.; Frick, M.; Heymer, B.; Schutte, S.; Wernik, S.; Schumann, H. J. Org. Chem. 1997, 62, 8681-8686. doi:10.1021/jo970822n

22. Dabrowski, J. A.; Villaume, M. T.; Hoveyda, A. H. Angew. Chem., Int. Ed. 2013, 52, 8156-8159. doi:10.1002/anie.201304035

23. Gurung, S. K.; Thapa, S.; Vangala, A. S.; Giri, R. Org. Lett. 2013, 15, 5378-5381. doi:10.1021/ol402701x

24. Thapa, S.; Gurung, S. K.; Dickie, D. A.; Giri, R. Angew. Chem., Int. Ed. 2014, 53, 11620-11624. doi:10.1002/anie.201407586

25. Gurung, S. K.; Thapa, S.; Kafle, A.; Dickie, D. A.; Giri, R. Org. Lett. 2014, 16, 1264-1267. doi:10.1021/ol500310u

26. Thapa, S.; Basnet, P.; Gurung, S. K.; Giri, R. Chem. Commun. 2015, 51, 4009-4012. doi:10.1039/C5CC00116A

27. Thapa, S.; Kafle, A.; Gurung, S. K.; Montoya, A.; Riedel, P.; Giri, R. Angew. Chem., Int. Ed. 2015, 54, 8236-8240. doi:10.1002/anie.201502379

28. Thathagar, M. B.; Beckers, J.; Rothenberg, G. J. Am. Chem. Soc. 2002, 124, 11858-11859. doi:10.1021/ja027716+

29. Li, J.-H.; Li, J.-L.; Wang, D.-P.; Pi, S.-F.; Xie, Y.-X.; Zhang, M.-B.; Hu, X.-C. J. Org. Chem. 2007, 72, 2053-2057. doi:10.1021/jo0623742

30. Zhou, Y.; You, W.; Smith, K. B.; Brown, M. K. Angew. Chem., Int. Ed. 2014, 53, 3475-3479. doi:10.1002/anie.201310275

31. You, W.; Brown, M. K. J. Am. Chem. Soc. 2014, 136, 14730-14733. doi:10.1021/ja509056j

32. Ito, H.; Sensui, H.-o.; Arimoto, K.; Miura, K.; Hosomi, A. Chem. Lett. 1997, 26, 639-640. doi:10.1246/cl.1997.639

33. Cornelissen, L.; Lefrancq, M.; Riant, O. Org. Lett. 2014, 16, 3024-3027. doi:10.1021/ol501140p

34. Yang, C.-T.; Zhang, Z.-Q.; Liang, J.; Liu, J.-H.; Lu, X.-Y.; Chen, H.-H.; Liu, L. J. Am. Chem. Soc. 2012, 134, 11124-11127. doi:10.1021/ja304848n

35. Terao, J.; Todo, H.; Begum, S. A.; Kuniyasu, H.; Kambe, N. Angew. Chem., Int. Ed. 2007, 46, 2086-2089. doi:10.1002/anie.200603451

36. Cahiez, G.; Gager, O.; Buendia, J. Angew. Chem., Int. Ed. 2010, 49, 1278-1281. doi:10.1002/anie.200905816

37. Mohapatra, S.; Bandyopadhyay, A.; Barma, D. K.; Capdevila, J. H.; Falck, J. R. Org. Lett. 2003, 5, 4759-4762. doi:10.1021/ol035458v

38. Allred, G. D.; Liebeskind, L. S. J. Am. Chem. Soc. 1996, 118, 2748-2749. doi:10.1021/ja9541239

39. Li, J.-H.; Tang, B.-X.; Tao, L.-M.; Xie, Y.-X.; Liang, Y.; Zhang, M.-B. J. Org. Chem. 2006, 71, 7488-7490. doi:10.1021/jo061220j

40. Thapa, S.; Shrestha, B.; Gurung, S. K.; Giri, R. Org. Biomol. Chem. 2015, 13, 4816-4827. doi:10.1039/C5OB00200A

41. Beletskaya, I. P.; Cheprakov, A. V. Coord. Chem. Rev. 2004, 248, 2337-2364. doi:10.1016/j.ccr.2004.09.014

42. Yang, C.-T.; Zhang, Z.-Q.; Liu, Y.-C.; Liu, L. Angew. Chem., Int. Ed. 2011, 50, 3904-3907. doi:10.1002/anie.201008007 
43. Cornelissen, L.; Cirriez, V.; Vercruysse, S.; Riant, O. Chem. Commun. 2014, 50, 8018-8020. doi:10.1039/c4cc02923b

44. The reaction of $\mathrm{Ph}_{3} \mathrm{Al}$ with 1-iodoocctane using the Schlenk technique afforded cross-coupled product 3 in $72 \%$ yield.

45. Cross-coupling of electron-deficient $\left(4-\mathrm{FC}_{6} \mathrm{H}_{4}\right)_{3} \mathrm{Al}$ with benzyl bromide afforded the product only in $18 \%$ yield.

46. The conversion of bromoolefins is generally over $90 \%$. The low to moderate yields of the cross-coupled products could result from protodebromination of the bromoolefins.

47. Giri, R.; Hartwig, J. F. J. Am. Chem. Soc. 2010, 132, 15860-15863. doi:10.1021/ja105695s

48. Tye, J. W.; Weng, Z.; Giri, R.; Hartwig, J. F. Angew. Chem., Int. Ed. 2010, 49, 2185-2189. doi:10.1002/anie.200902245

49. Tye, J. W.; Weng, Z.; Johns, A. M.; Incarvito, C. D.; Hartwig, J. F. J. Am. Chem. Soc. 2008, 130, 9971-9983. doi:10.1021/ja076668w

50. Strieter, E. R.; Bhayana, B.; Buchwald, S. L. J. Am. Chem. Soc. 2009, 131, 78-88. doi:10.1021/ja0781893

51. Do, H.-Q.; Khan, R. M. K.; Daugulis, O. J. Am. Chem. Soc. 2008, 130, 15185-15192. doi:10.1021/ja805688p

52. Schiefer, M.; Hatop, H.; Roesky, H. W.; Schmidt, H.-G.; Noltemeyer, M. Organometallics 2002, 21, 1300-1303. doi:10.1021/om010690v

53. Pour, N.; Gofer, Y.; Major, D. T.; Keinan-Adamsky, K.; Gottlieb, H. E.; Aurbach, D. Organometallics 2013, 32, 3165-3173. doi:10.1021/om300865a

54. Pour, N.; Gofer, Y.; Major, D. T.; Aurbach, D. J. Am. Chem. Soc. 2011, 133, 6270-6278. doi:10.1021/ja1098512

55. Damrauer, R.; Krempp, M.; Damrauer, N. H.; Schmidt, M. W.; Gordon, M. S. J. Am. Chem. Soc. 1993, 115, 5218-5226. doi:10.1021/ja00065a038

56. Krieck, S.; Görls, H.; Westerhausen, M. Organometallics 2008, 27, 5052-5057. doi:10.1021/om800509p

57. We analyzed a mixture of $\mathrm{Ph}_{3} \mathrm{Al}$ and $\mathrm{LiCl}$ using ${ }^{27} \mathrm{Al}$ NMR. No significant change was observed in the ${ }^{27} \mathrm{AI}$ NMR spectra. However, due to the strong Lewis acidic nature of $\mathrm{Ph}_{3} \mathrm{Al}$, formation of a small amount of $\left[\mathrm{Ph}_{3} \mathrm{AlCl}\right]^{-}[\mathrm{Li}]^{+}$cannot be ruled out without further experiments.

58. Dyer, P. W.; Fawcett, J.; Hanton, M. J. Organometallics 2008, 27, 5082-5087. doi:10.1021/om8005933

59. Brown, H. C.; Grayson, M. J. Am. Chem. Soc. 1953, 75, 20-24. doi:10.1021/ja01097a006

60. Periasamy, M.; Seenivasaperumal, M.; Padmaja, M.; Rao, V. D. ARKIVOC 2004, No. viii, 4-11.

61. Lindner, R.; van den Bosch, B.; Lutz, M.; Reek, J. N. H.; van der Vlugt, J. I. Organometallics 2011, 30, 499-510. doi:10.1021/om100804k

62. Breitenfeld, J.; Wodrich, M. D.; Hu, X. Organometallics 2014, 33, 5708-5715. doi:10.1021/om500506y

63. Uemura, M.; Yorimitsu, H.; Oshima, K. Chem. Commun. 2006, 4726-4728. doi:10.1039/b612173j

64. Di Franco, T.; Boutin, N.; Hu, X. Synthesis 2013, 45, 2949-2958. doi:10.1055/s-0033-1338544

65. Maruyama, K.; Noguchi-Yachide, T.; Sugita, K.; Hashimoto, Y.; Ishikawa, M. Bioorg. Med. Chem. Lett. 2010, 20, 6661-6666. doi:10.1016/j.bmcl.2010.09.011

66. Lipshutz, B. H.; Blomgren, P. A.; Kim, S.-K. Tetrahedron Lett. 1999, 40, 197-200. doi:10.1016/S0040-4039(98)02271-0

67. Liu, J.-H.; Yang, C.-T.; Lu, X.-Y.; Zhang, Z.-Q.; Xu, L.; Cui, M.; Lu, X.; Xiao, B.; Fu, Y.; Liu, L. Chem. - Eur. J. 2014, 20, 15334-15338. doi:10.1002/chem.201405223
68. Kori, M.; Hamamura, K.; Fuse, H.; Yamamoto, T. JP Patent JP2002/000532, 2002; p 748.

69. Dunsford, J. J.; Clark, E. R.; Ingleson, M. J. Angew. Chem., Int. Ed. 2015, 54, 5688-5692. doi:10.1002/anie.201411403

70. Tobisu, M.; Takahira, T.; Chatani, N. Org. Lett. 2015, 17, 4352-4355. doi:10.1021/acs.orglett.5b02200

71. Ellis-Davies, G. C. R.; Gilbert, A.; Heath, P.; Lane, J. C.; Warrington, J. V.; Westover, D. L. J. Chem. Soc., Perkin Trans. 2 1984, 1833-1841. doi:10.1039/p29840001833

\section{License and Terms}

This is an Open Access article under the terms of the Creative Commons Attribution License

(http://creativecommons.org/licenses/by/2.0), which permits unrestricted use, distribution, and reproduction in any medium, provided the original work is properly cited.

The license is subject to the Beilstein Journal of Organic Chemistry terms and conditions: (http://www.beilstein-journals.org/bjoc)

The definitive version of this article is the electronic one which can be found at: doi:10.3762/bjoc. 11.261 